\title{
HOMOMORPHISMS BETWEEN GENERALIZED VERMA MODULES
}

BY

BRIAN D. BOE ${ }^{1}$

\begin{abstract}
Let $\mathfrak{g}$ be a finite-dimensional complex semisimple Lie algebra and $\mathfrak{p}$ a parabolic subalgebra. The first result is a necessary and sufficient condition, in the spirit of the Bernstein-Gelfand-Gelfand theorem on Verma modules, for Lepowsky's "standard map" between two generalized Verma modules for $g$ to be zero. The main result gives a complete description of all homomorphisms between the generalized Verma modules induced from one-dimensional $\mathfrak{p}$-modules, in the "hermitian symmetric" situation.
\end{abstract}

1. Introduction. Let $\mathfrak{g}$ be a finite-dimensional complex semisimple Lie algebra and $\mathfrak{p}$ a parabolic subalgebra. A generalized Verma module (GVM) is a left $\mathrm{g}$-module $\mathscr{U}(\mathfrak{g}) \otimes_{\mathscr{U}(\mathfrak{p})} E$, where $E$ is a finite-dimensional irreducible $\mathfrak{p}$-module. A study of the g-homomorphisms between GVM's was begun by J. Lepowsky [10-12] and the purpose of the present work is to continue this study.

Suppose that $G$ is a real Lie group with complexified Lie algebra $g$ and $P$ a parabolic subgroup corresponding to $\mathfrak{p}$. There is a close connection between the representations of $G$ induced from finite-dimensional $P$-modules and the contragredient duals of algebraically induced modules for g (i.e. GVM's). For example, $P$ might be a "minimal" parabolic subgroup, and the representations of $G$ the nonunitary principal series. Homomorphisms between generalized Verma modules give rise, upon dualizing, to differential operators intertwining the representations of $G$.

In the main situation discussed in the present paper, this duality can be seen quite concretely. Suppose that $G / K$ is a hermitian symmetric space, where $K$ is a maximal compact subgroup. Take $\mathfrak{p}$ to be the associated parabolic subalgebra of $g$ (cf. §4). Denote by $r$ and $\mathfrak{u}^{+}$the reductive and nilpotent parts of $\mathfrak{p}$, and let $\mathfrak{u}^{-}$be the opposite nilradical. Then $\mathfrak{u}^{-}$is abelian. By the Poincaré-Birkhoff-Witt theorem, a homomorphism between generalized Verma modules is given by a certain matrix with entries from $\mathscr{U}\left(\mathfrak{u}^{-}\right) \simeq \mathscr{S}\left(\mathfrak{u}^{-}\right)$. Replacing each $x \in \mathfrak{u}^{-}$by $\partial / \partial x$ and transposing the matrix, gives the associated differential operator.

Received by the editors June 19, 1983 and, in revised form, July 5, 1984.

1980 Mathematics Subject Classification. Primary 17B10; Secondary 22E47.

Key words and phrases. Generalized Verma module, homomorphism, standard map, hermitian symmetric space, semi-invariant.

${ }^{1}$ Partially supported by Yale University Graduate Fellowship and N.S.E.R.C. (Canada) Postgraduate Scholarship. 
The $g$-module homomorphisms between (ordinary) Verma modules (where $\mathfrak{p}$ is a Borel subalgebra), have been completely characterized by Verma and BernsteinGelfand-Gelfand (B-G-G): all nonzero maps are injective, the space of homomorphisms is at most one-dimensional, and there is a necessary and sufficient condition for a nonzero map to exist (cf. $[1,2,5,17])$. However, in the case of generalized Verma modules, there are nontrivial homomorphisms which are not injective. Because of this, the argument used to prove multiplicity one for Verma modules does not carry over to GVM's. On the other hand, no example has been found to support the alternative conclusion, and it is one of the main unsolved problems to show that the space of maps is always at most one-dimensional (cf. [13]). ${ }^{2}$

Generalized Verma modules are highest weight modules, and hence are quotients of Verma modules. The existence of a nonzero map between GVM's implies containment of the corresponding Verma modules; conversely, such an inclusion induces a map between the GVM's, called the "standard map" [9]. But the standard map may be zero, and even when this happens, there might be some other "nonstandard" map. Thus the theory of Verma modules is not particularly useful in determining all homomorphisms between GVM's. Nevertheless, the standard maps can be described completely. Theorem 3.3 gives necessary and sufficient conditions (in the spirit of the B-G-G theorem) for the standard map to be nonzero.

The problem, then, is to understand the nonstandard maps. One tool which can be used is invariant theory. Suppose $E_{1}$ and $E_{2}$ are finite-dimensional irreducible $\mathrm{r}$-modules. Every $\mathrm{r}$-module homomorphism from $\mathscr{U}(\mathfrak{g}) \otimes_{\mathscr{U}(\mathfrak{p})} E_{1}$ to $\mathscr{U}(\mathfrak{g}) \otimes_{\mathscr{U}(\mathfrak{p})} E_{2}$ is determined by an $\mathfrak{r}$-invariant element of $\mathscr{U}\left(\mathfrak{u}^{-}\right) \otimes_{C} E_{1}^{*} \otimes_{C} E_{2}$. In general this tensor product is very difficult to decompose; however, when $E_{1}$ and $E_{2}$ are one-dimensional (the "scalar" case), one need only determine the r-semi-invariants in $\mathscr{U}\left(\mathfrak{u}^{-}\right)$. In the hermitian symmetric context described above, this has been done by $W$. Schmid [16]. Our main result is a complete characterization of when such an $r$-map is actually a g-map (Theorem 4.4). It should be noted that the proof given here is based on a result of $\mathrm{N}$. Wallach [18], and is different from the one in the author's Ph. D. thesis [3], which involved case-by-case computations.

In $\S 2$, we introduce the notation used in the remainder of the paper. $\$ 3$ is a discussion of standard maps, and $\S 4$ is devoted to the proof of Theorem 4.4.

This article is a summary of work done for my thesis at Yale University. I wish to thank my advisor, Gregg Zuckerman, for his guidance and encouragement.

2. Notation and preliminaries. Let $\mathfrak{g}$ be a finite-dimensional complex semisimple Lie algebra, $\mathfrak{h}$ a Cartan subalgebra, $\Delta \subset \mathfrak{h} *$ the set of roots of $\mathfrak{g}$ with respect to $\mathfrak{h}$, $\Delta^{+} \subset \Delta$ a positive system, and $\alpha_{1}, \ldots, \alpha$, the corresponding simple roots. Set $\rho$ $=\frac{1}{2} \sum_{\alpha \in \Delta^{+}} \alpha$. Define subalgebras

$$
\mathfrak{n}^{+}=\sum_{\alpha \in \Delta^{+}} \mathfrak{g}_{\alpha}, \quad \mathfrak{n}^{-}=\sum_{\alpha \in \Delta^{+}} \mathfrak{g}_{-\alpha}, \quad \text { and } \quad \mathfrak{b}=\mathfrak{h} \oplus \mathfrak{n}^{+}
$$

\footnotetext{
2ADDED IN PROOF. R. Irving has recently discovered a pair of generalized Verma modules (having singular infinitesimal character) for which the space of a-homomorphisms is two-dimensional.
} 
Denote by $($,$) the Killing form on \mathfrak{g}$, and also the form on $\mathfrak{h}^{*}$ induced by it. For $\alpha \in \Delta^{+}$, let $h_{\alpha}$ be the unique element of $\left[g_{\alpha}, g_{\alpha}\right]$ such that $\alpha\left(h_{\alpha}\right)=2$. We will write $h_{i}$ for $h_{\alpha_{i}}, 1 \leqslant i \leqslant l$. Also, let $s_{\alpha}$ be the reflection in the root $\alpha$. We have the Weyl group $W$ of $\mathfrak{g}$ with respect to $\mathfrak{h}$, and the length function $l(\cdots)$ on $W$. For $w$, $w^{\prime} \in W$, write $w \rightarrow w^{\prime}$ if there exists $\gamma \in \Delta^{+}$such that $w=s_{\gamma} w^{\prime}$ and $l(w)=l\left(w^{\prime}\right)+$ 1 ; the Bruhat order $\leqslant$ is defined to be the transitive closure of $\rightarrow$. Denote by $P^{+}$ the set of regular dominant integral weights of $\mathrm{g}$. For $\alpha$ a simple root, let $\omega_{\alpha}$ be the corresponding fundamental weight.

If $\lambda \in \mathfrak{h}^{*}$, we denote by $\mathbf{C}_{\lambda}$ the one-dimensional $\mathfrak{h}$-module on which $\mathfrak{h}$ acts by the form $\lambda$, and $\mathrm{n}^{+}$acts trivially. Define the Verma module

$$
M(\lambda)=\mathscr{U}(\mathfrak{g}) \otimes_{\mathscr{U}(\mathfrak{h})} \mathbf{C}_{\lambda-\rho} .
$$

Recall that $M(\lambda)$ has a unique irreducible quotient $L(\lambda)$, and admits an infinitesimal character $\Theta_{\lambda}$ for the center $\mathscr{Z}(\mathfrak{g})$ of $\mathscr{U}(\mathfrak{g})$; furthermore, $\Theta_{\lambda}=\Theta_{\mu}$ if and only if $\mu \in W \cdot \lambda$.

We shall now describe the construction of generalized Verma modules (GVM's). Because we need a rather explicit description of the parabolic subalgebras of $g$ containing the Borel subalgebra $\mathfrak{b}$, we must introduce some additional notation.

Let $S$ be any subset of $\{1, \ldots, l\}, \mathfrak{h}_{S}$ the (complex) span of the $h_{i}$ with $i \in S$, and $\mathfrak{g}_{S}$ the subalgebra of $\mathfrak{g}$ generated by $\mathfrak{h}_{S}$ and the $\mathfrak{g}_{ \pm \alpha_{i}}$ with $i \in S$. Set $\Delta_{S}=\Delta \cap$ $\sum_{i \in S} \mathbf{Z} \alpha_{i}, \Delta_{S}^{+}=\Delta^{+} \cap \Delta_{S}, \mathfrak{n}_{S}^{+}=\sum_{\alpha \in \Delta_{S}^{+}} \mathfrak{g}_{\alpha}$ and $\mathfrak{n}_{S}^{-}=\sum_{\alpha \in \Delta_{S}^{+}} \mathfrak{g}_{-\alpha}$. Then $\mathfrak{g}_{S}$ is a semisimple Lie algebra with Cartan subalgebra $\mathfrak{h}_{S}$, root system $\Delta_{S}$, positive roots $\Delta_{S}^{+}$and simple roots $\left\{\alpha_{i} \mid i \in S\right\}$ (when these elements of $\mathfrak{h}^{*}$ are restricted to $\mathfrak{h}_{S}$ ); we have $\mathfrak{g}_{S}=\mathfrak{n}_{S}^{-} \oplus \mathfrak{h}_{S} \oplus \mathfrak{n}_{S}^{+}$.

Define $\mathfrak{u}^{+}=\sum_{\alpha \in \Delta^{+} \backslash \Delta_{s}^{+}} \mathfrak{g}_{\alpha}, \mathfrak{u}^{-}=\sum_{\alpha \in \Delta^{+} \backslash \Delta_{s}^{+}} \mathfrak{g}_{-\alpha}, \mathfrak{r}=\mathfrak{h}+\mathfrak{g}_{S}$ and $\mathfrak{p} \equiv \mathfrak{p}_{S}=\mathfrak{r} \oplus \mathfrak{u}^{+}$. Then $\mathfrak{u}^{+}$and $\mathfrak{u}^{-}$are nilpotent subalgebras of $\mathfrak{n}^{+}$and $\mathfrak{n}^{-}$, respectively, and $\mathfrak{r}$ is a reductive subalgebra of $\mathfrak{g}$ with derived algebra $\mathfrak{g}_{S}$ and center $z(\mathfrak{r}) \subset \mathfrak{h}$. Since $\left[r, \mathfrak{u}^{+}\right] \subset \mathfrak{u}^{+}, \mathfrak{p}_{S}$ is a subalgebra of $g$ : it is the most general parabolic subalgebra containing the Borel $\mathfrak{b}$. If $S=\varnothing, \mathfrak{p}=\mathfrak{b}$; if $S=\{1, \ldots, l\}, \mathfrak{p}=\mathfrak{g}$. When $|S|=l-1$, we say that $\mathfrak{p}$ is a "maximal parabolic".

We note the following decompositions:

$$
\begin{gathered}
\mathfrak{g}=\mathfrak{u}^{-} \oplus \mathfrak{p}, \quad \mathfrak{r}=\mathfrak{g}_{S} \oplus z(\mathfrak{r}), \quad \mathfrak{h}=\mathfrak{h}_{S} \oplus \dot{z}(\mathfrak{r}), \\
\mathfrak{n}^{+}=\mathfrak{n}_{S}^{+} \oplus \mathfrak{u}^{+}, \quad \mathfrak{n}^{-}=\mathfrak{n}_{S}^{-} \oplus \mathfrak{u}^{-} .
\end{gathered}
$$

Set $\rho_{S}=\frac{1}{2} \sum_{\alpha \in \Delta_{S}^{+}} \alpha, \rho^{S}=\frac{1}{2} \sum_{\alpha \in \Delta^{+} \backslash \Delta_{S}^{+}} \alpha$. Then $\rho=\rho_{S}+\rho^{S}$. We tomark, for later use, that $\rho^{S} \mid \mathfrak{h}_{S}=0$ while $\rho^{S} \not \equiv 0$ (if $|S| \neq l$ ).

Let $P_{S}^{+}=\left\{\lambda \in \mathfrak{h}^{*} \mid \lambda\left(h_{i}\right) \in \mathbf{N}\right.$, all $\left.i \in s\right\}$. Then $P_{S}^{+}$parametrizes the set of (isomorphism classes of) finite-dimensional irreducible $r$-modules which remain irreducible under $\mathrm{g}_{S}$ : to $\lambda \in P_{S}^{+}$we associate the $\mathrm{r}$-module $E(\boldsymbol{\lambda})$ with highest weight $\lambda-\rho$ (relative to $\mathfrak{g}_{S}, \mathfrak{h}_{S}$, and $\mathfrak{n}_{S}^{+}$). We make $E(\lambda)$ a $\mathfrak{p}$-module by letting $\mathfrak{u}^{+}$act trivially. Define the generalized Verma module

$$
M_{S}(\lambda)=\mathscr{U}(\mathfrak{g}) \otimes_{\mathscr{U}_{\left(\mathfrak{p}_{S}\right)}} E(\lambda) .
$$

When $S=\varnothing, M_{S}(\lambda)$ is just the Verma module $M(\lambda)$; if $|S|=l$, then $\lambda \in P^{+}$and $M_{S}(\lambda)=L(\lambda)$. 
The basic properties of generalized Verma modules can be found in [5 or 9]; we recall here only that $M_{S}(\lambda)$ is a quotient of $M(\lambda)$.

To conclude this section, we define the subset $W^{S}$ of $W$ which parametrizes the generalized Verma modules having a given regular integral infinitesimal character. Let $W_{S}$ be the subgroup of $W$ generated by the $s_{i} \equiv s_{\alpha_{i}}$ with $i \in S$. When restricted to $\mathfrak{h}_{S}^{*}, W_{S}$ is just $W\left(\mathfrak{g}_{S}, \mathfrak{h}_{S}\right)$. Put

$$
W^{S}=\left\{w \in W \mid l\left(s_{i} w\right)=l(w)+1, \text { all } i \in S\right\},
$$

the set of minimal length right coset representatives of $W_{S}$ in $W$. (Note that some authors use left coset representatives.) Another characterization of $W^{S}$, which follows from [7, Corollary $10.2 \mathrm{C}]$, is

$$
W^{S}=\left\{w \in W \mid w^{-1} \Delta_{S}^{+} \subset \Delta^{+}\right\} .
$$

This description leads readily to the following proposition.

2.4. Proposition. Let $\lambda \in P^{+}$. Then $w \lambda \in P_{S}^{+}$if and only if $w \in W^{S}$.

Thus the GVM's having infinitesimal character $\Theta_{\lambda}$ are precisely the $M_{S}(w \lambda)$ with $w \in W^{S}$.

3. Standard maps. Let $\lambda, \mu \in P_{S}^{+}$, and suppose there is a nonzero g-map from $M_{S}(\lambda)$ to $M_{S}(\mu)$. Then $L(\lambda)$ is a composition factor of $M_{S}(\mu)$, hence of $M(\mu)$. Now by the B-G-G theorem, $M(\lambda) \subset M(\mu)$. Thus a necessary condition for the existence of a map between generalized Verma modules is containment of the corresponding Verma modules. Conversely, suppose there is a nonzero map $f: M(\lambda) \rightarrow M(\mu)$, with $\lambda$ and $\mu$ as above. Denote by $K(\lambda)$ the kernel of the projection $M(\lambda) \rightarrow M_{S}(\lambda)$. Then Lepowsky has shown [9, Proposition 3.1] that $f(K(\lambda)) \subset K(\mu)$, and hence $f$ induces a g-module map $\hat{f}: M_{S}(\lambda) \rightarrow M_{S}(\mu)$.

3.1. Definition (Lepowsky). The map $\hat{f}$ is called the standard map from $M_{S}(\lambda)$ to $M_{S}(\mu)$.

By the uniqueness of embeddings of Verma modules, the standard map is unique up to scalar multiples. At first sight, then, there appears to be an exact correlation between the existence of maps between GVM's and the existence of maps between the corresponding Verma modules. Unfortunately, the standard map can be zero. On the other hand, even when the standard map is zero, a nonzero homomorphism may exist. We shall call such maps "nonstandard". The author has discovered many examples of nonstandard maps, some of which are obtained from well-known differential operators such as the Laplacian, the Dirac and the Hodge star operators. For a more complete discussion, the reader is referred to [3].

The existence of nonstandard maps appears to make the theory of Verma modules relatively useless in the study of general homomorphisms between GVM's. However, the situation is much improved if we restrict our attention to standard maps.

3.2. Definition (B-G-G). Let $\lambda, \mu \in \mathfrak{h}^{*}$ and $\gamma_{1}, \ldots, \gamma_{r} \in \Delta^{+}$. Set $\mu_{0}=\mu$ and $\mu_{i}=s_{\gamma_{i}} \cdots s_{\gamma_{1}} \mu, 1 \leqslant i \leqslant r$. We say that the sequence $\left(\gamma_{1}, \ldots, \gamma_{r}\right)$ links $\mu$ to $\lambda$ (and that $\mu$ is linked to $\lambda$ ) if

(i) $\mu_{r}=\lambda$, and

(ii) $\mu_{i}=\mu_{i-1}-n_{i} \gamma_{i}$ with $n_{i} \in \mathbf{Z}_{+}, 1 \leqslant i \leqslant r$. 
The following theorem is a refinement of Lepowsky's work.

3.3. TheOREM. Let $\lambda, \mu \in P_{S}^{+}$, and assume that $M(\lambda) \subset M(\mu)$. The following are equivalent (notation as in Definition 3.2):

(i) The standard map $M_{S}(\lambda) \rightarrow M_{S}(\mu)$ is zero.

(ii) There exists a sequence $\left(\gamma_{1}, \ldots, \gamma_{r}\right)$ which links $\mu$ to $\lambda$, with $\mu_{1} \notin P_{S}^{+}$.

(iii) There exists a sequence $\left(\gamma_{1}, \ldots, \gamma_{r}\right)$ which links $\mu$ to $\lambda$, and an integer $i$, $1 \leqslant i \leqslant r$, with $\mu_{i} \notin P_{S}^{+}$.

Proof. (i) $\Rightarrow$ (ii). Assume that the standard map $M_{S}(\lambda) \rightarrow M_{S}(\mu)$ is zero. Then by [9, Proposition 3.3], $M(\lambda) \subset M\left(s_{j} \mu\right)$ for some $j \in S$. Hence (by B-G-G) there is a sequence $\left(\gamma_{2}, \ldots, \gamma_{r}\right)$ of positive roots linking $s_{j} \mu$ to $\lambda$. Put $\gamma_{1}=\alpha_{j}$. Then $\mu\left(h_{\gamma_{1}}\right) \in \mathbf{N}$ since $\mu \in P_{S}^{+}$, and $\left(s_{j} \mu\right)\left(h_{j}\right)=-\mu\left(h_{j}\right)$, so $s_{j} \mu \notin P_{S}^{+}$. Thus $\left(\gamma_{1}, \ldots, \gamma_{r}\right)$ satisfies (ii).

(ii) $\Rightarrow$ (iii). Obvious.

(iii) $\Rightarrow$ (i). Assume (iii). Then we have the commutative diagram

\begin{tabular}{|c|c|c|}
\hline \multicolumn{3}{|c|}{$M\left(\mu_{i}\right)$} \\
\hline & $g \nearrow$ & $\searrow h$ \\
\hline$M(\lambda)$ & $\vec{f}$ & $M(\mu)$ \\
\hline$\pi_{\lambda} \downarrow$ & & $\downarrow \pi_{\mu}$ \\
\hline$M_{S}(\lambda)$ & $\overrightarrow{\hat{f}}$ & $M_{S}(\mu)$ \\
\hline
\end{tabular}

where $\pi_{\lambda}$ and $\pi_{\mu}$ are projections, and $\hat{f}$ is the standard map. But since $\mu_{i} \notin P_{S}^{+}$, $\pi_{\mu} \circ h=0$ by [9, Proposition 3.1]. Hence $\hat{f} \circ \pi_{\lambda}=\pi_{\mu} \circ f=\pi_{\mu} \circ h \circ g=0$. But $\pi_{\lambda}$ is surjective, so $\hat{f}=0$. Q.E.D.

In the regular integral case, the theorem can be stated in terms of the Bruhat order.

3.4. Corollary. Let $\lambda \in P^{+}$and $w, w^{\prime} \in W^{S}$, with $w \leqslant w^{\prime}$. The following are equivalent:

(i) The standard map $M_{S}(w \lambda) \rightarrow M_{S}\left(w^{\prime} \lambda\right)$ is zero.

(ii) There exists $w_{1} \in W \backslash W^{S}$ such that $w \leqslant w_{1} \rightarrow w^{\prime}$.

(iii) There exists $w_{1} \in W \backslash W^{S}$ such that $w \leqslant w_{1} \leqslant w^{\prime}$.

3.5. Remark. The implication (ii) $\Rightarrow$ (i) is Proposition 3.9 of [9], while the equivalence of (ii) and (iii) also follows from Lemma 3.3 of [4].

The above result is most useful when applied to the following situation.

3.6. Corollary. Let $\lambda, w, w^{\prime}$ be as above. Then the standard map $M_{S}(w \lambda) \rightarrow$ $M_{S}\left(w^{\prime} \lambda\right)$ is nonzero if there exist distinct $w_{1}, w_{2} \in W^{S}$ with $w \rightarrow w_{1} \rightarrow w^{\prime}$ and $w \rightarrow w_{2} \rightarrow w^{\prime}$.

Proof. This is an easy consequence of Corollary 3.4(iii) and [1, Lemma 10.3].

4. Scalar maps: the hermitian symmetric case. Many of the results which have been obtained concerning generalized Verma modules have pertained only to those induced from one-dimensional $p$-modules. Thus, we make the following definition. 
4.1. Definition. A generalized Verma module $\mathscr{U}(\mathfrak{g}) \otimes_{\mathscr{U}(\mathfrak{p})} E$, with $E$ a one-dimensional $\mathfrak{p}$-module, is called a scalar GVM. A g-homomorphism between scalar GVM's is called a scalar map.

We shall determine all scalar maps in the "hermitian symmetric" situation, in the following sense.

Let $G$ be a simple real Lie group, $K$ a maximal compact subgroup, and assume that $G / K$ is a hermitian symmetric space. Let $g$ and be the corresponding complexified Lie algebras, and $\mathfrak{g}=\mathfrak{t} \oplus \mathfrak{q}$ a Cartan decomposition. If $\mathfrak{h}$ is a maximal abelian subalgebra of $\mathfrak{f}$, then $\mathfrak{h}$ is a Cartan subalgebra of $\mathfrak{g}$. By choosing an ordering of the roots of $\mathfrak{h}$ in $\mathfrak{g}$, we obtain a decomposition $\mathfrak{q}=\mathfrak{q}^{+} \oplus \mathfrak{q}^{-}$. Then $\mathfrak{p}=\mathfrak{t} \oplus \mathfrak{q}^{+}$is a parabolic subalgebra of $\mathfrak{g}$. We shall call such a parabolic $\mathfrak{p}$ a hermitian symmetric parabolic subalgebra. The roots whose spaces lie in $\mathfrak{f}$ (resp. q) are called compact (resp. noncompact) roots.

The hermitian symmetric parabolic subalgebras of a complex simple Lie algebra $g$ are characterized by the condition that the nilradical $\mathfrak{u}^{+}$of $\mathfrak{p}$ (and hence also $\mathfrak{u}^{-}$) be abelian. For the remainder of this section, we shall assume that this condition holds.

Now, suppose $E$ and $E^{\prime}$ are one-dimensional p-modules, having nonzero elements $e$ and $e^{\prime}$, and highest weights $\lambda$ and $\lambda^{\prime}$. Every g-map

$$
\phi: \mathscr{U}(\mathrm{g}) \otimes_{\mathscr{U}(\mathfrak{p})} E^{\prime} \rightarrow \mathscr{U}(\mathfrak{g}) \otimes_{\mathscr{U}(\mathfrak{p})} E
$$

is determined by the image $\phi\left(1 \otimes e^{\prime}\right)=u \otimes e$, where we may assume that $u \in \mathscr{U}\left(\mathfrak{u}^{-}\right)$. Since $g_{S}$ is semisimple, and $E$ and $E^{\prime}$ are one-dimensional, they are trivial as $\mathfrak{g}_{S}$-modules. It follows that $u \in \mathscr{U}\left(\mathfrak{u}^{-}\right)^{\mathfrak{g} s}$, the space of $\mathfrak{g}_{S^{-}}$-invariant vectors in $\mathscr{U}\left(\mathfrak{u}^{-}\right)$. Similarly, by using the fact that $\phi$ is an $\mathfrak{h}$-module map, one finds that $u$ is a weight vector of weight $\lambda^{\prime}-\lambda$. Hence $u$ is an $r$-semi-invariant in $\mathscr{U}\left(\mathfrak{u}^{-}\right)$.

Thus, a first step in determining the maps between scalar GVM's is to identify the $r$-semi-invariants. Denote by $\alpha$ the unique simple noncompact root. We introduce the usual order $\leqslant$ on $\mathfrak{h}^{*}: \lambda \leqslant \mu$ if $\mu-\lambda$ is a nonnegative integral linear combination of the simple roots. Let $\gamma_{1}, \ldots, \gamma_{r}$ be the maximal family of mutually orthogonal noncompact roots, as constructed by Harish-Chandra [6]. In particular, $\gamma_{1}=\alpha$ and $\gamma_{1}<\gamma_{2}<\cdots<\gamma_{r}$. Moore has shown [15] that $\left(\gamma_{i}, \gamma_{i}\right)=\left(\gamma_{j}, \gamma_{j}\right)$ for $1 \leqslant i, j \leqslant r$. Set $\mathfrak{h}^{-}=\sum_{i=1}^{r} \mathrm{C} h_{\gamma_{i}}$.

Following Wallach [18], we set $\mu_{j}=-\left(\gamma_{1}+\cdots+\gamma_{j}\right), 1 \leqslant j \leqslant r$. Then, by Schmid's theorem [16], the r-highest weight vectors of $\mathscr{U}\left(\mathfrak{u}^{-}\right)$are in one-to-one correspondence with the weights $\sum_{i=1}^{r} n_{i} \mu_{i}, n_{i} \in \mathbf{Z}_{+}$. Let $u_{j}$ be a nonzero element of $\mathscr{U}\left(\mathfrak{u}^{-}\right)^{\mathfrak{n}_{S}^{+}}$having weight $\mu_{j}$. Then $\mathscr{U}\left(\mathfrak{u}^{-}\right)^{\mathfrak{n}_{S}^{+}}$is the subalgebra of $\mathscr{U}\left(\mathfrak{u}^{-}\right)$generated by $u_{1}, \ldots, u_{r}$.

4.2. Proposition. $\mathscr{U}\left(\mathfrak{u}^{-}\right)^{\mathfrak{A} s}$ is either $\mathbf{C}\left[u_{r}\right]$ or $\mathbf{C}$.

Proof. Let $u \in \mathscr{U}\left(\mathfrak{u}^{-}\right)$be a weight vector for $\mathfrak{h}$, say of weight $\lambda$. If $u$ is $\mathrm{g}_{S}$-invariant, then by Schmid's theorem,

$$
\lambda=-\sum_{k=1}^{r} m_{k} \gamma_{k}, \quad m_{1} \geqslant m_{2} \geqslant \cdots \geqslant m_{r} .
$$

Now $\mathscr{U}(\mathrm{r}) u$ is a finite-dimensional irreducible $r$-module; thus, $u$ is $\mathfrak{g}_{s}$-invariant if and only if $\lambda \mid \mathfrak{h}_{S} \equiv$ 
Moore [15, Theorem 2] has shown that given $1 \leqslant j<i \leqslant r$, there exists $\beta \in \Delta_{S}^{+}$ such that $\beta \mid \mathfrak{h}^{-}=\frac{1}{2}\left(\gamma_{i}-\gamma_{j}\right)$. Then

$$
0=\lambda\left(h_{\beta}\right)=\frac{2}{(\beta, \beta)}\left(\frac{1}{2}\left(\gamma_{i}-\gamma_{j}\right),-\sum_{k=1}^{r} m_{k} \gamma_{k}\right)=\frac{\left(\gamma_{j}, \gamma_{j}\right)}{(\beta, \beta)}\left(m_{j}-m_{i}\right) \text {. }
$$

Hence $m_{i}=m_{j}$, and $\lambda=-m \sum_{k=1}^{r} \gamma_{k}=m \mu_{r}$, where $m$ is the common value of the $m_{k}$ 's. Thus $u=u_{r}^{m}$ (up to scalar multiple).

In case (a) of Moore's result, every element $\beta$ of $\Delta_{S}^{+}$has the property that $\beta \mid \mathfrak{h}^{-}$ $=\frac{1}{2}\left(\gamma_{i}-\gamma_{j}\right)$ for some $1 \leqslant j<i \leqslant r$. In this case, we have $\mathscr{U}\left(\mathfrak{u}^{-}\right)^{\mathfrak{g}_{s}}=\mathbf{C}\left[u_{r}\right]$. However, in case (b), there also exists $\beta \in \Delta_{S}^{+}$with $\beta \mid \mathfrak{h}^{-}=-\frac{1}{2} \gamma_{r}$. Then

$$
0=\lambda\left(h_{\beta}\right)=\frac{\left(\gamma_{r}, \gamma_{r}\right)}{(\beta, \beta)} m
$$

forcing $m=0$. Thus in this case $\mathscr{U}\left(\mathfrak{u}^{-}\right)^{\mathfrak{g} s}=$ C. Q.E.D.

4.3. REmARKs. 1. It follows from the proof that the cases $\mathscr{U}\left(\mathfrak{u}^{-}\right)=\mathbf{C}\left[u_{r}\right]$ or $\mathbf{C}$ correspond to cases (a) and (b), respectively, of Moore's theorem [15, Theorem 2]. A. Koranyi and J. Wolf [8] proved that condition (a) is equivalent to the condition that $G / K$ be a tube domain. Wolf [19] showed that this, in turn, is necessary and sufficient for the existence of a nontrivial $\mathfrak{g}_{S^{-}}$-invariant in $\mathscr{U}\left(\mathfrak{u}^{-}\right)$.

2. R. Lipsman and J. Wolf [14] have proved that $\mathscr{U}\left(\mathfrak{u}^{-}\right)$has a nontrivial $\mathrm{g}_{S}$-invariant precisely when the longest element of $W$ sends $\alpha$ to $-\alpha$.

4.4. TheOREM. Let $\mathfrak{g}$ be a simple Lie algebra, $\mathfrak{p}=\mathfrak{p}_{S}$ a hermitian symmetric parabolic subalgebra, and $\alpha$ the unique simple noncompact root. Assume that $\mathscr{U}\left(\mathfrak{u}^{-}\right)^{\mathfrak{g}_{s}}$ $\neq$ C. Let $\lambda, \lambda^{\prime} \in \mathfrak{h}^{*}$ be such that $M_{s}(\lambda+\rho)$ and $M_{S}\left(\lambda^{\prime}+\rho\right)$ are scalar GVM's. Then $\operatorname{Hom}_{\mathscr{U}(\mathfrak{g})}\left(M_{S}\left(\lambda^{\prime}+\rho\right), M_{S}(\lambda+\rho)\right)$ is nonzero if and only if either $\lambda=\lambda^{\prime}$, or $\lambda=$ $\left(k-\rho^{S}\left(h_{\alpha}\right)\right) \omega_{\alpha}$ and $\lambda^{\prime}=\left(-k-\rho^{S}\left(h_{\alpha}\right)\right) \omega_{\alpha}$ for some $k \in \mathbf{N}$.

Proof. Clearly we may assume $\lambda \neq \lambda^{\prime}$. Since $E(\lambda+\rho)$ is a one-dimensional $\mathrm{r}$-module, it is trivial as a $\mathrm{g}_{S}$-module. Hence $\lambda\left(h_{i}\right)=0$ for all $i \in S$. Thus $\lambda=z \omega_{\alpha}$ for some $z \in \mathbf{C}$. Let $e, e^{\prime}$ be nonzero elements of $E(\lambda+\rho), E\left(\lambda^{\prime}+\rho\right)$. If $\phi$ : $M_{S}\left(\lambda^{\prime}+\rho\right) \rightarrow M_{S}(\lambda+\rho)$ is a nonzero r-module map, then $\phi\left(1 \otimes e^{\prime}\right)=u \otimes e$ for some $u \in \mathscr{U}\left(\mathfrak{u}^{-}\right)^{\mathfrak{g}_{s}}$ having weight $\lambda^{\prime}-\lambda$.

Suppose we show that $x \cdot(u \otimes e)=0$ for some nonzero $x \in \mathfrak{u}^{+}$. Since $\mathfrak{u}^{+}$is abelian, it is irreducible as an $\left(\operatorname{adg}_{S}\right)$-module. Now for $g \in g_{s}$,

$$
[g, x] \cdot(u \otimes e)=g \cdot x \cdot(u \otimes e)-x \cdot g \cdot(u \otimes e)=0
$$

it follows that $u \otimes e$ is $\mathfrak{u}^{+}$-invariant. Hence, by the universal property of induced modules, $\phi$ is in fact a g-map.

Now, by Proposition 4.2, $u=u_{r}^{k}$ for some $k \in \mathbf{N}$, and Wallach has computed the commutator $\left[E_{\gamma_{r}}, u_{r}\right]$, where $E_{\gamma_{r}}$ spans $\mathfrak{g}_{\gamma_{r}} \subset \mathfrak{u}^{+}\left[18\right.$, Lemma 5.9]. Set $x=E_{\gamma_{r}}$ and $u=u_{r}$. Then

$$
\begin{aligned}
x \cdot\left(u^{k} \otimes e\right) & =\sum_{i=1}^{k} u^{i-1}[x, u] u^{k-i} \otimes e \\
& =\sum_{i=1}^{k} u^{i-1}\left\{\frac{1}{2}\left(\gamma_{r}, \gamma_{r}\right) u_{r-1} h_{\gamma_{r}}+\frac{1}{4}\left(\gamma_{r}, \gamma_{r}\right) c_{r} u_{r-1}+\sum_{\beta \in \Delta_{S}^{+}} x_{\beta} e_{\beta}\right\} u^{k-i} \otimes e,
\end{aligned}
$$


where $x_{\beta} \in \mathscr{U}\left(\mathfrak{u}^{-}\right), e_{\beta} \in \mathfrak{g}_{\beta}$ and $c_{r}=\#\left\{\beta \in \Delta_{S}^{+}|\beta| \mathfrak{h}^{-}=\frac{1}{2}\left(\gamma_{r}-\gamma_{j}\right)\right.$ for some $\left.j<r\right\}$. Since $\mathfrak{g}_{S} \cdot u=0$ and $\mathfrak{g}_{S} \cdot e=0$, the third term vanishes. An easy computation, using the fact that $\alpha=\gamma_{1}$ and $\left(\gamma_{1}, \gamma_{1}\right)=\left(\gamma_{r}, \gamma_{r}\right)$, shows that $\omega_{\alpha}\left(h_{\gamma_{r}}\right)=1$. Also, since $\mu_{r}=-\left(\gamma_{1}+\cdots+\gamma_{r}\right)$ and $\left(\gamma_{i}, \gamma_{j}\right)=0$ for $i \neq j, \mu_{r}\left(h_{\gamma_{i}}\right)=-2$ for $1 \leqslant i \leqslant r$. Hence

$$
\begin{aligned}
x \cdot\left(u^{k} \otimes e\right)= & \sum_{i=1}^{k} u^{i-1}\left\{\frac{1}{2}\left(\gamma_{r}, \gamma_{r}\right) u_{r-1}\left((k-i) \mu_{r}\left(h_{\gamma_{r}}\right)+z \omega_{\alpha}\left(h_{\gamma_{r}}\right)\right)\right. \\
& \left.\quad+\frac{1}{4}\left(\gamma_{r}, \gamma_{r}\right) c_{r} u_{r-1}\right\} u^{k-i} \otimes e \\
= & \frac{1}{4} k\left(\gamma_{r}, \gamma_{r}\right)\left\{-2(k-1)+2 z+c_{r}\right\} u_{r-1} u^{k-1} \otimes e .
\end{aligned}
$$

This is zero precisely when $z=k-1-\frac{1}{2} c_{r}$. Since by hypothesis, $g$ satisfies condition (a) of Moore's theorem (cf. Remark 4.3.1), a result of Harish-Chandra [6, Lemma 18] implies that $\frac{1}{2} c_{r}=\rho^{S}\left(h_{\gamma_{r}}\right)-1$. Now it follows from $\S 2$ that $\rho^{S}$ is a multiple of $\omega_{\alpha}$; since $\omega_{\alpha}\left(h_{\gamma_{r}}\right)=\omega_{\alpha}\left(h_{\alpha}\right)$, we have $\frac{1}{2} c_{r}=\rho^{S}\left(h_{\alpha}\right)-1$. Hence $x \cdot\left(u^{k} \otimes\right.$ $e)=0$ if and only if $z=k \cdot-\rho^{S}\left(h_{\alpha}\right)$.

It remains only to compute $\lambda^{\prime}$. But $\lambda^{\prime}=\lambda+k \mu_{r}$ and $\mu_{r} \mid \mathfrak{h}_{S}=0$, so $\mu_{r}$ is also a multiple of $\omega_{\alpha}$. Since $\mu_{r}\left(h_{\alpha}\right)=-2, \mu_{r}=-2 \omega_{\alpha}$ and $\lambda^{\prime}=\left(-k-\rho^{S}\left(h_{\alpha}\right)\right) \omega_{\alpha}$. Q.E.D.

4.5. Remarks. 1. Lepowsky has proved [13] that the space of maps between two scalar generalized Verma modules is at most one-dimensional; the same result-in the hermitian symmetric case-also follows from the above proof.

2. Note that the proof also describes explicitly all nontrivial scalar maps.

3. One can show, using Remark 4.3.2, that $w^{0}(\lambda+\rho)=\lambda^{\prime}+\rho$ for $\lambda \neq \lambda^{\prime}$ as in the theorem (where $w^{0}$ is the longest element of $W^{S}$ ). Thus $\operatorname{Hom}_{\mathscr{U}(\mathfrak{g})}\left(M_{S}\left(w^{0} \rho\right), M_{S}(\rho)\right)$ $\neq 0$ if and only if $\rho^{S}\left(h_{\alpha}\right) \in \mathbf{N}$ (under the hypotheses of the theorem). The same conclusion applies to $\operatorname{Hom}_{\mathscr{U}(\mathfrak{g})}\left(M_{S}\left(w^{0} \mu\right), M_{S}(\mu)\right)$ for any $\mu \in P^{+}$, by the Translation Principle of Jantzen and Zuckerman.

\section{REFERENCES}

1. I. N. Bernstein, I. M. Gelfand and S. I. Gelfand, Differential operators on the base affine space and a study of (1-modules, Lie Groups and their Representations (I. M. Gelfand, ed.), Wiley, New York, 1975. pp. $21-64$.

2. Structure of representations generated by vectors of highest weight, Functional Anal. Appl. 5 (1971), 1-8.

3. B. Boe, Homomorphisms between generalized Verma modules, Ph. D. thesis, Yale University, 1982.

4. V. V. Deodhar, Some characterizations of the Bruhat ordering on a Coxeter group and determination of the relative Mobius function, Invent. Math. 39 (1977), 187-198.

5. J. Dixmier, Enveloping algebras, North-Holland, Amsterdam and New York, 1977.

6. Harish-Chandra, Representations of semisimple Lie groups. VI, Amer. J. Math. 78 (1956), 564-628.

7. J. Humphreys, Introduction to Lie algebras and representation theory, Springer-Verlag, New York, 1972.

8. A. Koranyi and J. A. Wolf, Realization of hermitian symmetric spaces as generalized half-planes, Ann. of Math. (2) 81 (1965), 265-288.

9. J. Lepowsky, A generalization of the Bernstein-Gelfand-Gelfand resolution, J. Algebra 49 (1977), 496-511.

10. Conical vectors in induced modules, Trans. Amer. Math. Soc. 208 (1975), 219-272.

11. Existence of conical vectors in induced modules, Ann. of Math. (2) 102 (1975), 17-40.

12. On the uniqueness of conical vectors, Proc. Amer. Math. Soc. 57 (1976), 217-220.

13. Uniqueness of embeddings of certain induced modules, Proc. Amer. Math. Soc. 56 (1976), $55-58$. 
14. R. L. Lipsman and J. A. Wolf, Canonical semi-invariants and the Plancherel formula for parabolic subgroups, Trans. Amer. Math. Soc. 269 (1982), 111-131.

15. C. C. Moore, Compactifications of symmetric spaces. II: the Cartan domains, Amer. J. Math. 86 (1964), 358-378.

16. W. Schmid, Die Randwerte holomorpher Functionen auf hermitesh symmetrischen Raumen, Invent. Math. 9 (1969), 61-80.

17. D. N. Verma, Structure of certain induced representations of complex semi-simple Lie algebras, $\mathrm{Ph} . \mathrm{D}$. thesis, Yale University, 1966.

18. N. R. Wallach, The analvtic continuation of the discrete series. II, Trans. Amer. Math. Soc. 251 (1979), 19-37.

19. J. A. Wolf, Classification and Fourier inversion for parabolic subgroups with square integrable nilradical, Mem. Amer. Math. Soc. No. 225 (1979).

Department of Mathematics, University of Utah, Salt Lake City, Utah 84112 